\title{
CONSTRUCCIONES A BASE DE TIERRA (EARTH-BASED CONSTRUCTION)
}

\author{
Marina Alvarez Alonso y M. a Jesús Guinea, Arquitectas* \\ Juan Díaz-Romeral, Aparejador* \\ 127-13
}

\section{RESUMEN}

Trabajo realizado y basado en las nuevas posibilidades de las construcciones con tierra, una vez considerado su interés en épocas anteriores y las nuevas posibilidades que ofrece ante la crítica impuesta por la crisis de la energía.

Se hacen consideraciones sobre las necesidades de vivienda previstas hasta el año 2000 y otros parámetros que vuelven a ser importantes al confrontarlos económicamente con los materiales industrializados, sistemas de construcción, soluciones orientadas a los países en vías de desarrollo, etc.

Continúa el trabajo con las primeras vías de experimentación práctica desarrolladas por el equipo de trabajo de viviendas de bajo coste orientadas a la búsqueda y promoción de recursos locales y abundantes como el material tierra, vías de experimentación que comienzan con el conocimiento por el equipo de técnicas y materiales durante el curso realizado en Ciadueña (Soria).

Se describen aspectos técnicos del material tierra desarrollados paralelamente a la experiencia práctica inicial y orientada a la elección idónea de los componentes, una vez confrontada la información bibliográfica relacionada con el tema.

Entre las consideraciones técnicas se llega a una exposición más exhaustiva sobre:

1) Las técnicas existentes: ladrillos de adobe, tierra apisonada (tapial), etc.

2) Porcentajes idóneos de los componentes para la elección de los materiales preferibles en cada una de las técnicas anteriores.

3) Consideraciones de los aspectos físicos y mecánicos de la maquinaria existente y en experimentación, así como de las posibilidades respecto a su puesta en obra, costes comparativos, etc.

4) Estudio de las posibilidades para mejorar las características del material tierra mediante diferentes técnicas de estabilización (compactación y correcciones granulométricas, estabilizantes fisicoquimicos, betunes, productos industriales, etc.).

Termina el artículo con una serie de conclusiones orientadas a disipar los posibles prejuicios existentes sobre las construcciones de tierra.

\section{Antecedentes}

El problema de la vivienda es uno de los más difíciles de resolver en los países en vías de desarro-

\footnotetext{
* Participantes en el Equipo de Investigación "Viviendas de muy bajo coste", que se desarrollará en el IET durante el trienio 1985-87.
}

\section{$S U M M A R Y$}

Work has been carried out based on the new possibilities of using earth in construction, after considering its interest in previous times and the new possibilities which it offers in the face of criticism imposed by the energy crisis.

Predicted housing needs up to the year 2000 have been considered along with other parameters which have once again grown in importance on being compared economically with industrialised materials, construction systems, solutions oriented towards the developing countries, etc.

Work continues along the first lines of practical experimentation, developed by the team working on low-cost housing oriented towards the search for and the promotion of abundant local resources such as earth, avenues of research whose starting-point involves the team becoming acquainted with techniques and materials during the course conducted in Ciadueña (Soria).

The technical aspects of earthen material are described: these have been developed along the same lines as initial practical experiments oriented towards the most suitable selection of its components, once the bibliographical data related to this subject have been collated.

Amongst the technical considerations, a more exhaustive exposition has been reached concerning:

1) Existing techniques: adobe bricks, packed earth (moulds for mudwalls), etc.

2) The most appropriate component percentages for the selection of. material considered to be preferable for each of the previous techniques.

3) Considerations of the physical and mechanical aspects of existing machinery and that undergoing testing, as well as the possibilities with regard to its being put into operation, comparative costs, etc.

4) The study of the possibilities for improving the characteristics of earthen material, by means of different stabilization techniques (making it compact, granulometric corrections, physicochemical stabilizers, bitumen, industrial products, etc.).

The article ends with a series of conclusions oriented towards dissipating possible prejudices which exist with regard to earthen constructions.

Ilo. El déficit habitacional crece de forma alarmante y la capacidad adquisitiva de los usuarios decrece. Así se llega a la necesidad de utilización de materiales locales tradicionales, abundantes y de bajo coste como el bambú, la madera, piedra o tierra, debido al aumento progresivo del valor de la energía y la escasez de materias primas. 
El cemento - material de construcción, en la actualidad, más importante - no es accesible a todo el mundo. De todos los materiales la tierra, al ser abundante, económica y relativamente fácil de poner en obra -en autoconstrucción - será la más utilizada. Por el contrario, tiene como inconveniente una gran sensibilidad al agua y perder en presencia de ésta todas las propiedades mecánicas. Este inconveniente puede ser evitado ya sea por estabilizadores, ya por dispositivos arquitectónicos o constructivos apropiados.

La arquitectura de tierra tiene un nuevo porvenir, tratándose de recuperar la más antigua técnica constructiva autóctona. Su utilización se remonta en la historia de la Humanidad al año 6000 a. C., aportando una tecnología a la vez popular y sabia. Pondremos nuestras miras en aquellas culturas que han sabido seguir elaborando sus construcciones en tierra como son las de Africa y Oriente Medio, con un saber hacer y saber vivir en las mismas.

Encontramos ejemplos de construcciones con tierra en Castilla (España), Rumania, Francia e incluso en países lluviosos como Gran Bretaña o Dinamarca. En Europa Occidental desapareció este arte a partir de los años 1920 a 1930, con la llegada del movimiento internacional, el cual rechazará la arquitectura de tierra para glorificar las cualidades de la industrialización masiva de la edificación, este abandono se debió a la aparición de materiales industrializados, a su precio relativamente bajo y a su facilidad de ejecución.

El interés por la construcción de tierra puede renacer, pues la crisis de la energía ha ímpuesto una nueva crítica de nuestra construcción y consumo, debiendo recurrir a los materiales que necesiten para su elaboración la menor energía posible. Los costes comparativos vuelven a ser parámetros importantes al confrontarlos económicamente con materiales como el hormigón o soluciones a base de bloques prefabricados.

La arquitectura de tierra en los años 80 es y será un campo de reflexión, experimentación y acción, y también un medio de abordar un diálogo nortesur entre países ricos y pobres.

En países en desarrollo, hasta ahora se ha intentado copiar modelos occidentales de construcción en hormigón por tendencias sicológicas, pero un sistema de hormigón en tierra estabilizada y compactada con encofrados metálicos demuestra estar bien situado para vencer estas reticencias hacia el "lado moderno".

\section{Vías de experimentación}

Nuestro interés por las construcciones en tierra se debe a estar actualmente en desarrollo el proyecto de investigación "Materiales, tecnologías y proto- tipos de viviendas de muy bajo costen (Soluciones para España y adecuación a condiciones, incluso extremas, del área Centro-Americana) en el IETCC y pertenecer al equipo de trabajo específicamente constituido para ello, encontrándose dentro de la fase de búsqueda de materiales y materias primas semielaboradas, que aporten bajas sensibles a los costes de utilización e incluso aceptando una cierta disminución cualitativa de los mismos.

Se trata, pues, de promover materias, procesos o empleos nuevos adaptados al marco ambiental fisico, económico y sociológico, con un mejor aprovechamiento de los recursos locales, regionales y nacionales.

La realización del primer Curso de Construcción de adobe y tapial llevado a cabo en Ciadueña (Soria) patrocinado por INTER-ACCION y dirigido por el arquitecto Erhard Rohmer, nos ofreció la oportunidad de dar un primer paso de toma de contacto en el conocimiento de técnicas y materiales, dirigido hacia una posterior investigación seria a medio plazo en el tema.

Fue un aprendizaje de las primitivas técnicas artesanales de realización de adobe y tapial con los utensilios tradicionales utilizados por el pueblo como adoberas, artesas, etc. (foto 1). La experiencia de la gente del lugar nos llevó a un sitio determinado, junto al río Duero, debido a la imperiosa necesidad del agua para encontrar buena tierra.

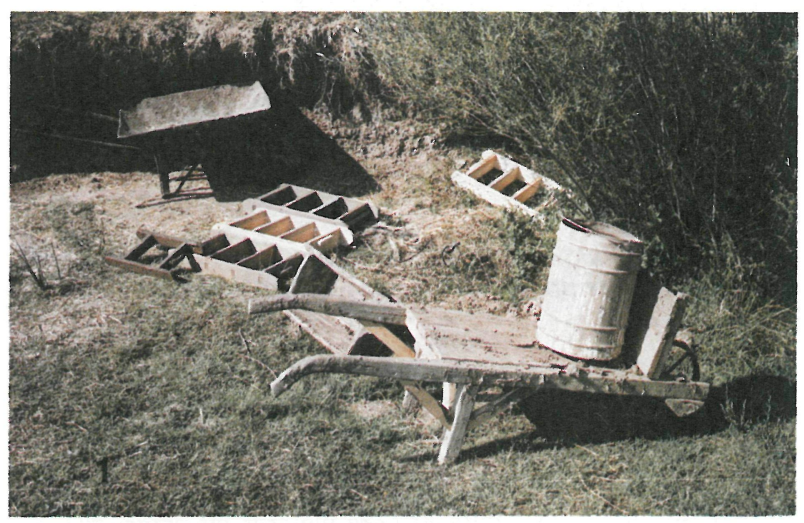

Utensilios empleados en la fabricación del adobe.

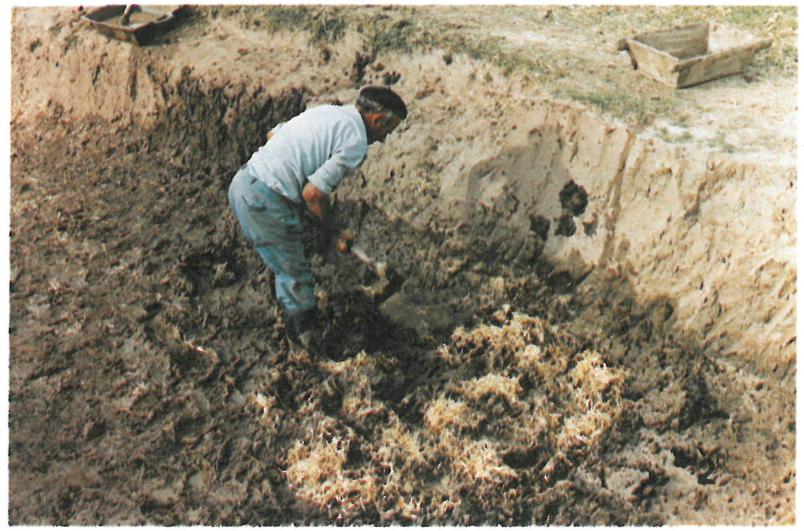

Pisado del barro con la paja hasta conseguir una homogeneidad. 
La primera fase, para la realización de adobe, es la preparación de la tierra estabilizándola con paja amasada con el barro, hasta que queda una mezcla compacta, uniforme y sin grumos (antes este procedimiento se realizaba "pisando"). (foto 2 ).

Después se rellenan los moldes (foto 3 ), que solian ser de madera $y$, en la actualidad, metálicos, siendo los ladrillos de dimensiones variables según los hábitos locales; éstos se dejan sobre el mismo terreno, más o menos Ilano, en estado plástico, no pudiéndolos mover hasta que no pasen de 3 a 5 días de secado. Tras este tiempo se ponen de canto con un secado complementario de 3 a 4 semanas (foto 4). Los adobes secos son

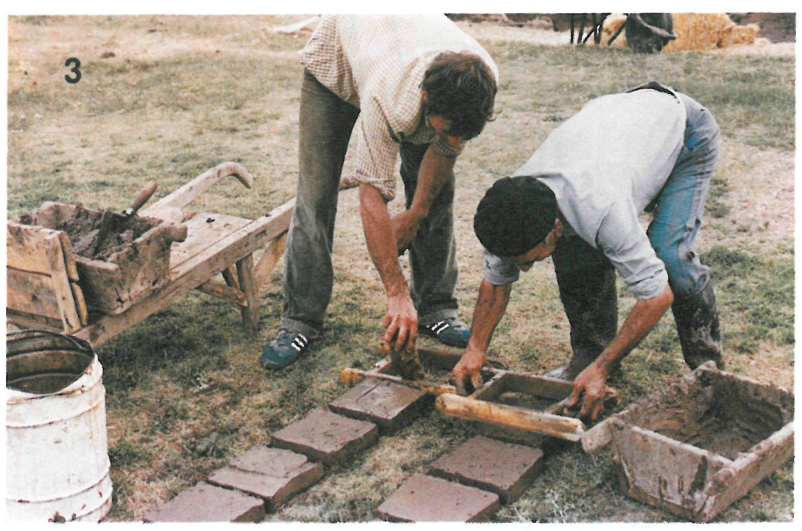

Las caras interiores de la adobera de madera se mojan y se rellena la misma del barro, evitando que se pegue.

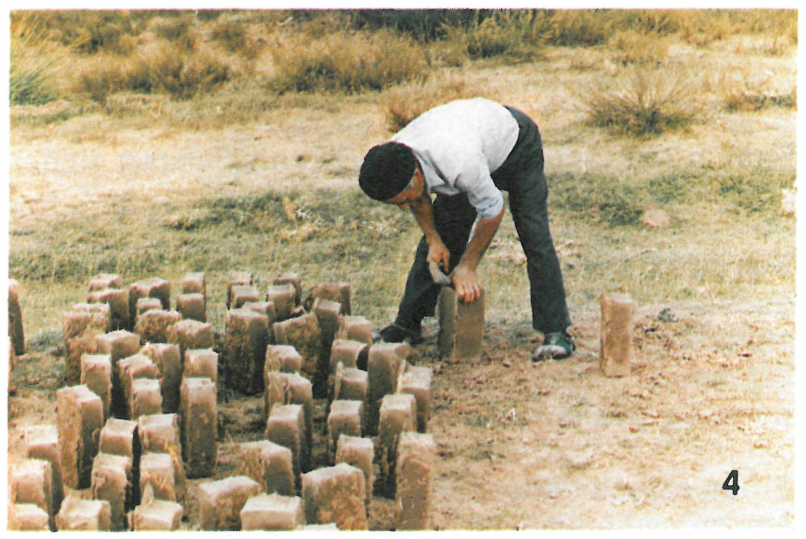

Corte de las aristas para regularizar el adobe.

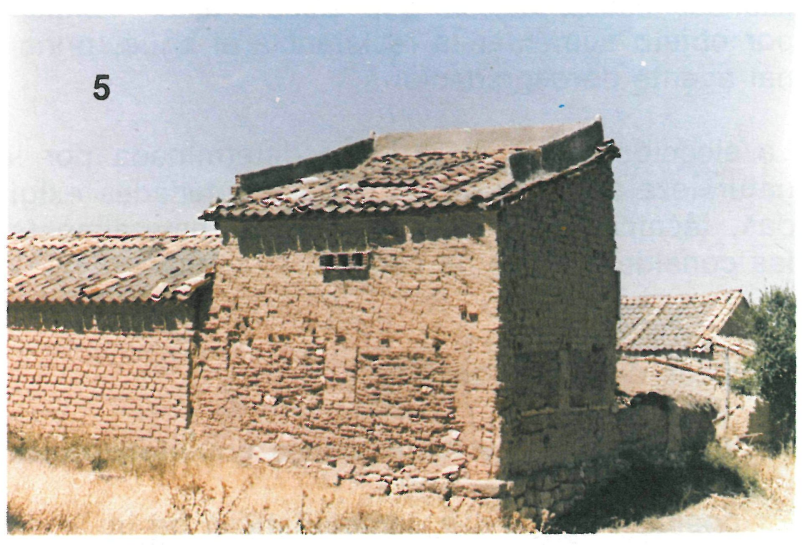

Palomar de Ciadueña construido con adobes. aparejados con ayuda de un mortero del mismo barro.

La elección del tipo de tierra generalmente ha venido transmitida por tradición de la experiencia popular, hasta encontrar la idónea, siendo evidente que este proceso debe ir acompañado de otra serie de consideraciones de carácter técnico, como ensayos granulométricos, sedimentométricos, determinación de los límites de Atterberg, ensayo de compactación (Proctor), etc.

\section{Aspectos técnicos}

Los suelos están esencialmente formados por granos sólidos de diferentes tamaños debido a la erosión de las rocas, distinguiendo los cantos, gravas, arenas, limos y arcillas. Los limos y las arcillas juegan el papel más importante en la ligazón de los suelos, siendo muy sensibles a la humedad, presentando retracciones al desecar e incrementos de volumen en presencia de agua, factores que habrá que tener en cuenta.

El suelo más favorable para la realización de adobes es el que contiene arena en un 55 a $75 \%$, limo del 10 al $28 \%$ y arcilla del 15 al $18 \%$.

En general, las construcciones en tierra sólo trabajan bien a compresión, y se necesita una buena distribución de cargas si existen varios niveles; poseen una gran inercia térmica; su precio es reducido $y$, en numerosos casos, aportan un mejor aislamiento que los bloques de hormigón. Es fundamental realizar una perfecta hermeticidad de revoques, techos y cimientos por su importante sensibilidad al agua.

La utilización del adobe ha sido frecuente en Europa, América, Africa y Oriente Medio, habiendo en la actualidad sistemas semiindustriales de producción en el sur de EE.UU.

\section{Otras Técnicas}

Otra de las técnicas utilizadas en las construcciones de tierra es el tapial. Consiste en apisonar la tierra por tongadas entre dos planchas de madera (foto 6). Cuando se ha terminado una sección de muro, se desplaza el encofrado hacia arriba o hacia un lado y se continúa la operación hasta su realización total. El apisonado puede hacerse manualmente con la ayuda de un pisón (foto 7) 0 utilizando una prensa mecánica. Las dimensiones más generalizadas del encofrado son de $300 \mathrm{~cm}$ de longitud, $90 \mathrm{~cm}$ de altura y por $50 \mathrm{~cm}$ de ancho. La mejor tierra para realizarlo es la que contiene de 0 al $15 \%$ de grava, 40 a $50 \%$ de arena, 20 a $35 \%$ de limos y del 15 al $25 \%$ de arcilla. Puede estabilizarse resultando entonces el hormigón de tierra estabilizado. Se utilizaba en Marruecos, Perú, Bolivia y España. 
La manufactura directa es otra técnica de utilizar la tierra. Consiste en moldearla en estado plástico y sin ayuda de encofrado. Se utiliza sobre todo en Africa Negra. El secado es a veces más lento y las resistencias mecánicas y a la intemperie son a menudo mediocres.

Después de definidas estas técnicas, es clara la necesidad de tender a una producción más industrializada, con una maquinaria adecuada para que el proceso sea más rápido y menos trabajoso. Esta necesidad se resuelve en parte con la fabri-

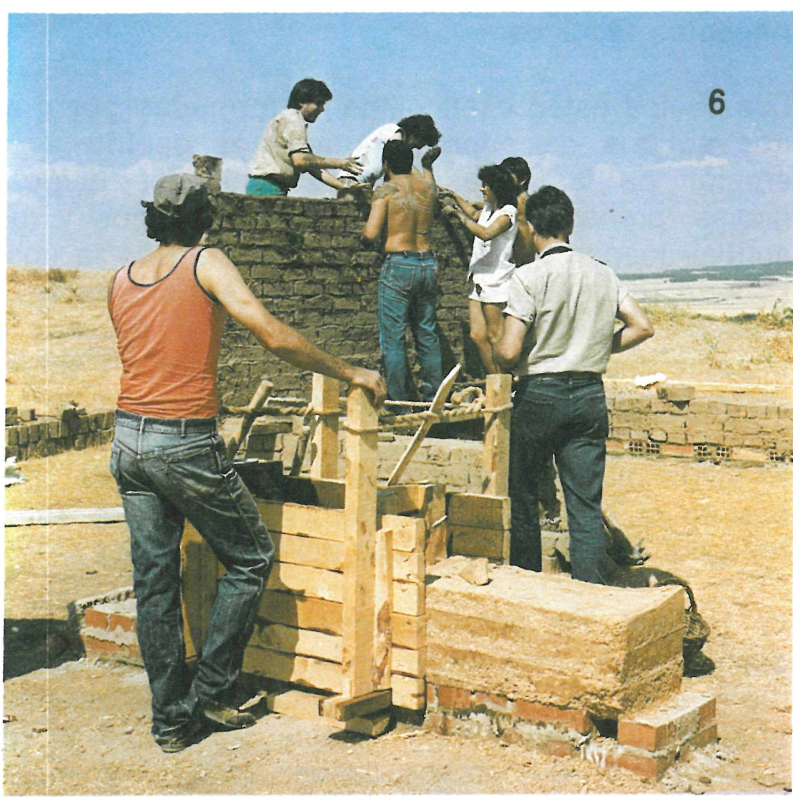

Formación del tapial por tongadas de tierra apisonada.

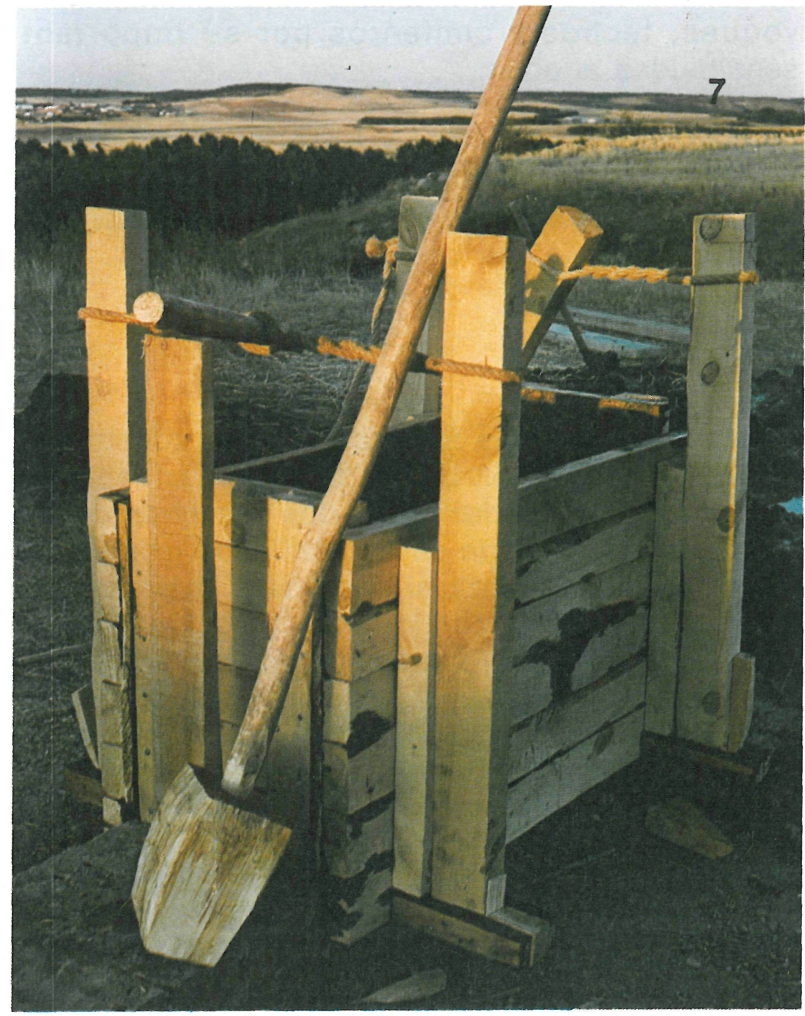

Encofrado de madera para el tapial con pisón manual. cación de bloques comprimidos, cuya técnica consiste en compactar en un molde una tierra relativamente seca cuyo contenido de agua es comparable a la que se utiliza en el tapial.

Existen prensas que permiten realizar los ladrillos comprimidos - pudiendo ser manuales - donde la

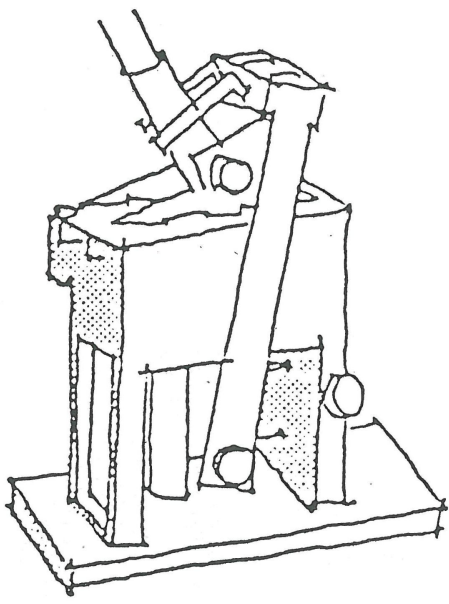

8

Prensa CINVA-RAM.

compactación se ayuda con un sistema de palanca, como la prensa CINVA-RAM (fig. 8) o la multibloc BREPAK, y también mecánicas, que permiten la fabricación de varios cientos de ladrillos al día, e incluso miles en los modelos más sofisticados.

Las presiones teóricas de fabricación varían de 20 a $200 \mathrm{~kg} / \mathrm{cm}^{2}$, según se utilice la prensa CinvaRam o maquinaria de mayor complejidad (hipercompresión).

Después del secado, los ladrillos se ponen en obra de la misma manera que los adobes, con almacenamiento inmediato. Las propiedades mecánicas son más elevadas y la estabilización de la tierra se puede realizar con cemento, cal o betún.

Si las características del material en cualquiera de los procedimientos no son convenientes, es necesario experimentar y mejorarlas, es decir, estabilizarlas; estabilización que puede tener también por objeto aumentar la resistencia al agua, principal agente de degradación.

La elección del sistema viene determinada por la naturaleza del material de base, propiedades exigidas, técnicas de puesta en obra y, naturalmente, las consideraciones de coste.

\section{Técnicas de estabilización}

\section{Compactación y correcciones granulométricas.}

Es posible mejorar las características de un suelo por simple compactación. Una gran energía de 


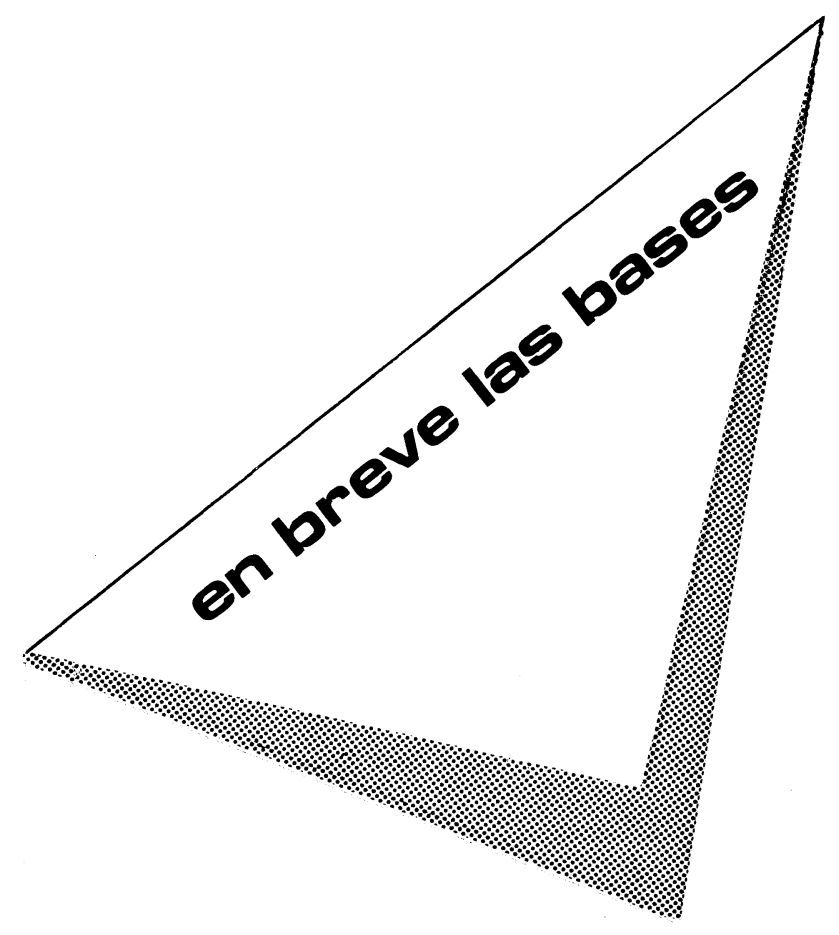

\section{II concurso}

Iberoamericano de informes

1986

compactación disminuye la cantidad de agua y trae consigo una mayor densidad seca y una resistencia mecánica más alta; aumenta el contacto entre las partículas y disminuye la macroporosidad del material.

Ciertos suelos, que poseen problemas por sus características granulométricas, se pueden modificar con la ayuda de un material de características diferentes; una tierra muy arenosa puede ser corregida con aporte de finos y otra más plástica con aporte de arena. Es posible ayudarse de curvas granulométricas para calcular las propiedades de una mezcla óptima.

\section{Estabilizantes fisicoquímicos.}

Los productos más utilizados para aumentar las propiedades de los materiales son: cementos, cales y betunes. Con el cemento se aumentan las resistencias mecánicas a compresión en estado seco y sobre todo en estado húmedo, se reducen las variaciones dimensionales y crece la resistencia a la erosión. Las tierras que se pueden estabilizar con cemento deben contener al menos un $10 \%$ de arcilla, así como arena y grava.

Las materias orgánicas y los sulfatos son desfavorables. Los contenidos máximos de cemento son del orden del $10 \%$ en masa, y los contenidos habituales se sitúan alrededor del $8 \%$. Los cementos utilizados son los portland con o sin adiciones secundarias.

La estabilización con cal: puede utilizarse cal viva o cal apagada $(\mathrm{CaO}, \mathrm{Ca}(\mathrm{OH}) 2)$. El desarrollo de las resistencias es más lento que con el cemento y se realiza por la reacción arcilla-cal, preferentemente en ambiente húmedo. La estabilización a base de cal conviene particularmente con tierra arcillosa. La cal se acomoda con materias orgánicas mejor que el cemento, siendo las cantidades habituales de cal entre el $3 \%$ y el $8 \%$ en masa.

Las mezclas cal-cemento son también utilizadas en algunos casos.

\section{Los betunes.}

La tercera posibilidad de estabilización la ofrecen los betunes. Estos productos son muy utilizados en la fabricación de adobes en EE.UU.

Se utilizan en forma de emulsiones en el agua y en forma de solución de disolventes (nafta y queroseno).
Los betunes aumentan la resistencia mecánica en seco, pero esta resistencia decrece rápidamente con cantidades muy elevadas. La absorción de agua se ralentiza fuertemente $y$, en consecuencia, la resistencia en estado húmedo se mantiene. Los contenidos en betún utilizados son entre el $2 \%$ y el $6 \%$ y los suelos más favorables son los plásticos.

\section{Otros productos.}

Son los productos naturales, tales como cenizas, gomas, aceites o productos industriales como los ácidos, resinas o silicatos. También se propone un cierto número de productos comerciales a base de aminas cuaternarias, aminas grasas o productos polímeros. Estos productos son todavía poco utilizados y sus características están aún por precisar.

A pesar del estabilizante experimentado es necesario hacer notar que jamás se obtendrán resultados espectaculares con una tierra nefasta.

De todas formas es necesario, para obtener buenos resultados, una proporción correcta del material estabilizado en particular a la hora de la mezcla y del curado.

\section{Conclusiones}

Dado que se estiman unas necesidades de unos 500 millones de viviendas, de aquí al año 2000 , la política oficial en los distintos países en vías de desarrollo, una vez respaldada la infraestructura inicial necesaria para la construcción en tierra de al menos un número rentable de viviendas relativamente estandarizadas, podría ser dejar paso poco a poco a las iniciativas individuales, y que tanto los organismos privados o de promoción de viviendas sociales se apropiaran de estos procedimientos.

Aunque las perspectivas políticas, industriales e incluso sicológicas, aparecen mucho más abiertas, los prejuicios sobre la construcción en tierra, así como el uso de la energía solar son grandes, sobre todo en las sociedades multinacionales, pues construir en tierra se logra con pequeñas y medianas industrias. Una combinación de los dos sistemas tierra-sol es importante para poder ahorrar hasta un $75 \%$ de las necesidades energéticas en climas donde el verano es caliente y el invierno riguroso. 